\title{
Application Research on Location Selection of Logistics Distribution Center Based on Immune optimization algorithm
}

\author{
SONG Qiang \\ School of Mechanical Engineering, Anyang Institute of Technology \\ e-mail: 13523323305@126.com
}

Key words: Logistics distribution center; Immune optimization algorithm; Location selection

\begin{abstract}
With logistics cost as objective function, the immune optimization algorithm was proposed to optimize the location selection of logistics distribution center. Feasibility analysis was conducted on the logistics demand points of 31 cities in China. Results indicated that the programming of the optimization algorithm based on Matlab was simple, with quick computing speed. It can solve the location problems with time restrictions as well as the traditional location problems without time restrictions. Therefore, such new algorithm is more effective than the gravity approach to solve the location problem of logistics center.
\end{abstract}

\section{Introduction}

With the rapid development of world economy and progress of modern science and technology, logistics industry, as an emerging service sector of national economy, is developing rapidly worldwide. Meanwhile, development of logistics industry has a huge influence on social production and management, people's livelihood and employment as well as the functions of government and legal system. Therefore, logistics are considered as the artery and foundation industry of national economic development. It is also vividly compared to the "accelerator" promoting economic development.

During the operation of logistics system, the task of distribution center is timely, accurate and economical distribution of goods and cargos based on users' individual requirements. As the bridge connecting suppliers with customers, the location selection of distribution center is of significant importance. It basically determines the distribution range and patterns of logistics, thus affecting the efficiency of logistics system. In addition, once the location of logistics center is determined, it is difficult to make changes. Therefore, the location of distribution center is of important theoretical and practical application significance. In general, the location model of logistics center is non-convex and non-smooth nonlinear programming model with complex constraints, belonging to NP-hard problem.

There are several methods to solve the problem of logistics center location, such as AHP, expert selection, comparative analysis, fuzzy evaluation and mixed integer programming method. AHP, expert selection, comparative analysis and fuzzy evaluation belong to qualitative analysis, with certain subjective factors. When faced with large-scale problem, the solution will become much difficult. Thus, there is an urgent requirement to find a convenient and efficient algorithm for the location selection of distribution center.

\section{Establishment of location selection models of logistics distribution center}

The location problem of distribution center can be described to select a certain number of locations (p) among limited positions (m). Through the establishment of distribution centers with reasonable scale, the goods of $\mathrm{n}$ distribution points can be supplied by these distribution centers. Meanwhile, such distribution centers should satisfy the premise of distribution requirements, with the lowest costs (including the construction and operation costs). Therefore, several assumptions have been made in the location model of logistics distribution center.

(1) The scale and capacity of distribution center satisfy the requirements of demand points, determined by the demand quantity within the radiation scope; 
(2) A demand point is supplied by only one distribution center;

(3) There is no need to consider the transportation costs between factory and distribution center;

Based on above assumptions, a location / distribution model is established as follows. Below the upper limit of distance, a distribution center should be selected from $\mathrm{n}$ demand points to deliver cargos to other demand points. Thus, the objective function is the minimum sum of the product of demand and distance from distribution center to demand points, and it is as follows.

$$
\min F=\sum_{J \in n} \sum_{j \in M_{1}} \varpi_{i j} d_{i j} Z_{i j}
$$

Constraint condition is

$$
\begin{aligned}
& \sum_{j \in M_{1}} Z_{i j}=1, i \in N \\
& Z_{i j} \leq h_{j}, i \leq N, j \leq M_{i} \\
& \sum_{J \in M_{I}} h_{j}=p \\
& \quad Z_{i j}, h_{j} \in\{0,1\}, i \in N, j \in M_{i} \\
& h_{i j} \leq s
\end{aligned}
$$

where $N=\{1,2, \cdots, n\}$ is the set of numbers of demand points; $M_{i}$ the set of alternative distribution centers with the distance less than s to demand points; $i \in N, M_{i} \subseteq N$; $w_{i}$ is the demand of distribution points; $d_{i j}$ the distance from demand point i to the nearest distribution center; $Z_{i j}$ a variable between $0-1$, representing the services demand and distribution relationship between users and logistics centers; $Z_{i j}=1$ the demand of demand point $\mathrm{j}$ is supplied by distribution point $\mathrm{j}$, otherwise $Z_{i j}=0 ; h_{j}$ a variable between $0-1$; when $h_{j}=1$, it means that point $\mathrm{j}$ has been selected as the distribution center; $\mathrm{s}$ the superior distance limit from new distribution center to the demand points within service range.

where Equation (1-2) ensures that each demand point is supplied by only a distribution center; Equation (1-3) ensures that the needs of demand point can only be supplied by the point set as the distribution center, namely there will not be customers in the point without distribution center. Equation (1-4) specifies the number of selected distribution centers is p; Equation (1-5) represents that $Z_{i j}$ and $h_{j}$ are variables between 0-1; Equation (1-6) ensures that the demand point is within the range that distribution center can reach.

\section{Simulation results and analysis}

To prove the feasibility and effectiveness of algorithm, the coordinates of 31 cities were collected in China. Table 3-1 shows the location of each user and their demand of materials. Such demand of materials is the result after the normalization process, not representing the actual value. Six of these cities were selected as the logistics distribution centers.

According to the location model of distribution center, the numerical example was solved following the steps of immune algorithm. Parameters of algorithm includes population size of 50, memory base capacity of 10 , iteration numbers of 100 , crossover probability of 0.5 , mutation probability of 0.4 and diversity assessment parameter of 0.95 , respectively. Then, results of solutions showed that the location of distribution center was [18 255279 14]. In this scenario, the sum of distance by making the demand of each demand point as weight was $5.68 \times 10^{5}$. 
Table 3-1 User's position and material demand

\begin{tabular}{|c|c|c||c|c|c||c|c|c|}
\hline $\mathrm{j}$ & $\left(U_{j}, V_{j}\right)$ & $b_{j}$ & $\mathrm{j}$ & $\left(U_{j}, V_{j}\right)$ & $b_{j}$ & $\mathrm{j}$ & $\left(U_{j}, V_{j}\right)$ & $b_{j}$ \\
\hline 1 & $(1304,2312)$ & 20 & 12 & $(2562,1756)$ & 40 & 23 & $(3429,1908)$ & 80 \\
\hline 2 & $(3639,1315)$ & 90 & 13 & $(2788,1491)$ & 40 & 24 & $(3507,2376)$ & 70 \\
\hline 3 & $(4177,2244)$ & 90 & 14 & $(2381,1676)$ & 40 & 25 & $(3394,2643)$ & 80 \\
\hline 4 & $(3712,1399)$ & 60 & 15 & $(1332,695)$ & 20 & 26 & $(3439,3201)$ & 40 \\
\hline 5 & $(3488,1535)$ & 70 & 16 & $(3715,1678)$ & 80 & 27 & $(2935,3240)$ & 40 \\
\hline 6 & $(3326,1556)$ & 70 & 17 & $(3918,2179)$ & 90 & 28 & $(3140,3550)$ & 60 \\
\hline 7 & $(3238,1229)$ & 40 & 18 & $(4061,2370)$ & 70 & 29 & $(2545,2357)$ & 70 \\
\hline 8 & $(4196,1044)$ & 90 & 19 & $(3780,2212)$ & 100 & 30 & $(2778,2826)$ & 50 \\
\hline 9 & $(4312,790)$ & 90 & 20 & $(3676,2578)$ & 50 & 31 & $(2370,2975)$ & 30 \\
\hline 10 & $(4386,570)$ & 70 & 21 & $(4029,2838)$ & 50 & & & \\
\hline 11 & $(3007,1970)$ & 60 & 22 & $(4263,2931)$ & 50 & & & \\
\hline
\end{tabular}

Fig.3-1 shows the convergence curve of immune algorithm, and Fig.3-2 shows the acquired location scheme of logistics distribution center.

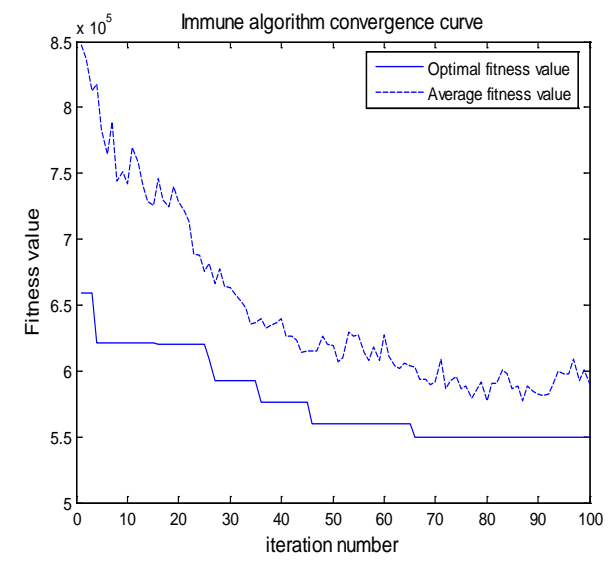

Fig.3 Convergence curve of immune algorithm

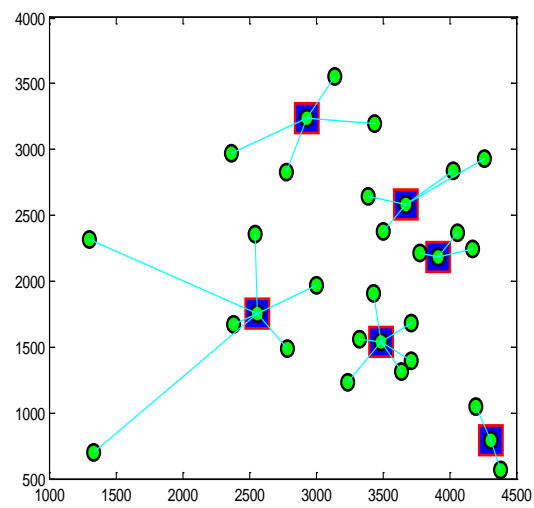

Fig.4 Location scheme of logistics distribution center

\section{Conclusions}

Based on mathematical model of location optimization for logistics distribution center, an immune optimization algorithm was constructed in the work to solve such problem. With the introduction of selective amplification of antibody clones and antibody inhibition, the algorithm was provided with strong optimization ability. Then, simulation was conducted on the location selection of logistics distribution center. Results showed that the optimal solution of location of logistics distribution center can easily be obtained by immune optimization algorithm.

\section{Acknowledgements}

First of all, thanks to the scientific research project of Henan Province(NO:16A510013).I would like to express my gratitude to all those who helped me during the writing of this thesis. I gratefully acknowledge the help of my supervisor, Mr. YANG Cheng-zhi, who has offered me valuable suggestions in the academic studies. In the preparation of the thesis, he has spent much time reading through each draft and provided me with inspiring advice. Without her patient instruction, insightful criticism and expert guidance, the completion of this thesis would not have been possible.I should finally like to express my gratitude to my beloved parents who have always been helping me out of difficulties and supporting without a word of complaint. 


\section{References}

[1].Imuune Computing Engineering [M], Beijing: Science Press, 2007.

[2]. Shi Feng, Wang Hui, et al. Thirty Cases Analysis of MATLAB Intelligent Algorithm [M], Beijing: Beihang University Press, 2011.7.

[3].Liu Bing, Artificial Immune Algorithm and Its Application [D], Chongqing: Chongqing University, 2004.

[4].Guo Li, Dynamic Location Research of Logistics Distribution Center under Random Demand [D], Chengdu: Southwest Jiaotong University, 2006.

[5].Zhou Meifang, Ye Hongtao, Location of Logistics Distribution Center Based on Immune Algorithm [J], Journal of Guangxi University of Technology, 2012, 23 (3): 77-79.

[6].YU Zong-yan, HAN Lian-tao.An Immune Optimization Algorithm with Particle Swarm and Its application[J], Computer Simulation,2008(12):111-121.

[7].zhu-hong ZHANG.Immune Optimization Algorithm for constrained nonlinear multiobjective Optimization problemes[J], Applied soft computing,2007(7):840-857.

[8].Zne-Jung Leea, , Chou-Yuan Leeb, Shun-Feng Su.An immunity-based ant colony optimization algorithm for solving weapon-target assignment problem[J], Applied Soft Computing2002(8), Pages 39-47.

[9].Xinchao Zhaoa, b, Boqian Songa, Panyu Huanga, Zichao Wena, Jialei Wengc, Yi Fan.An improved discrete immune optimization algorithm based on PSO for QoS-driven web service composition[J],Applied Soft Computing,2012(8): 2208-2216.

[10]LAN Fangpeng1,DUAN Fu2 1.Immune optimization algorithm for shipping arrangement of water transport of coal[J],Computer Engineering and Applications,2012(4):121-133.

[11]QIAN Shu-qu1,WU Hui-hong1.Dynamic Immune Optimization Algorithm and Its Application in Knapsack Problem[J],computer sen engineering,2011（20） : 265-277.

[12]ZHANG Zhu-Hong QIAN Shu-Qu (Department of Mathematics,College of Science,Guizhou University,Guiyang 550025);Adaptive Immune Algorithm and Its Track to Dynamic Function Optimization[J];Pattern Recognition and Artificial Intelligence;2007（1） : 121-127. 